\title{
Stimulus correlates of visual pattern discrimination by monkeys: Multidimensional analyses'
}

\author{
V. J. POLIDORA \\ UNIVERSITY OF WISCONSIN
}

Fourteen monkeys were presented with a series of simultaneous discrimination problems, each problem consisting of two visual metric pattems, and $S s^{\prime}$ performance was analyzed with respect to several physical dimensions of the patterns. Proficiency of discriminative performance was found to be directly and almost exclusively related to the extent to which the two patterns, when translated, had unique parts.

The primary goal of this series of experiments has been to determine the physical characteristics of visual metric patterns which determine the proficiency with which monkeys perform two-choice, simultaneous pattern discrimination problems. The stimuli used thus far in these experiments have been luminous patterns generated by lighting certain combinations of 12 square lights mounted in a 3 by 4 matrix (Webster, 1966, reviews other studies using similar patterns). Two such metric patterns were presented to learning setsophisticated monkeys for series of simultaneous discrimination problems, and Ss' percentage of correct responses on each problem has been assumed to be directly related to the extent to which the two patterns were discriminable.

The results of previous experiments have identified several characteristics of these visual metric patterns as being systematically related to discriminative performance by monkeys (Polidora \& Thompson, 1964; Polidora, 1965b; Polidora \& Thompson, 1965) and humans (Polidora, 1965a). The present experiment was designed (1) to determine in a single experiment the direct and interactive effects upon discriminative performance of each of four dimensions previously identified in separate experiments; (2) to determine whether the same results hold also for the larger and more useful population of patterns available from 16-light, 4 by 4 matrices; and (3) to permit multidimensional analyses of the data in order to evaluate the extent to which the dimensions under study, as well as others suggested in the literature, contribute uniquely to discriminability.

From a more general viewpoint the results of these experiments are relevant also to the problem of determining the physical characteristics of two or more patterns which make them discriminable (Hake, 1957; Silver, Landis, \& Messick, 1966; Stenson, 1966; Webster, 1966; Wohlwill, 1966), and they are therefore not necessarily relevant to the problem of psychophysical description of single patterns. Moreover, these investigations have been exclusively concerned with only one type of stimulus domain, visual metric patterns. The decision to work with this admittedly restricted stimulus domain was based upon its relatively limited dimensionality, the ease with which such experiments could be conducted, and the simplicity of specification of the patterns used. Furthermore, if the physical basis of discriminability of these simple patterns could be determined, the results should facilitate designing more critical experiments with more complexpatterns.

\section{Subjects}

\section{METHOD}

The Ss were eight male and six female rhesus monkeys (Macaca mulatta), 6 to 9 years of age. The experimental history of each $\mathrm{S}$ was identical (Harlow, Harlow, Rueping, \& Mason, 1960; Polidora \& Fletcher, 1964; Polidora \& Thompson, 1964; Polidora, 1965b; Polidora \& Thompson, 1965), and they were thus uniformly test-sophisticated. The Ss were first ranked on the basis of their total percentage correct response scores in the latter experiment, and two matched groups were obtained by randomly assigning one $S$ of each of the successive pairs of ranked Ss to Group A and the remaining $S$ to Group $B$. In each group there were four male and three female Ss.

\section{Apparafus}

An automated discrimination apparatus (for details see Polidora \& Main, 1963 and Polidora \& Thompson, 1965) consisting of three principal components was used. (1) An IBM 526 Printing Summary Punch (keypunch) programmed trials by reading prepunched program cards specifying the stimuli and reward contingency of each trial. The keypunch also recorded the discriminative responses on each trial into IBM punched cards, thus facilitating later computer analyses without intermediate data reduction. (2) A control console contained the necessary electronic components. (3) S's test compartment was in a remote, sound-attenuated experimental room, and it consisted of a 23-in. cubic restraining box and a work panel, both made of black, nonreflective acrylic plastic. Centered on the front wall of the box was a form-fitting fiberglass face mask with eyeholes and three vertical slots through which $S$ could extend its arms to touch the vertical work panel which was 11 in. from the mask eyeholes. The mask eyeholes and the vertical centers of the three stimulus displays on the work panel were 16 in. from the floor. The two outer displays were the only ones used in this experiment, and a reward receptacle was mounted immediately below each. Completely overlaying each 
display was a rigid piece of glass which had a transparent, distortion-free, conductive layer which was connected to one pole of a contact relay circuit. Ss performed discriminative responses by touching the glass of one display or the other, thus completing the circuit to the floor of the box (see Polidora, 1965c for details).

To initiate each trial $\mathrm{S}$ was required to position its head into the facemask, thereby interrupting the forehead-level photobeam and causing both displays to light simultaneously. After the mask response hadbeen maintained for $0.5 \mathrm{sec}$., a window-touching response to either display caused both displays to extinguish immediately, a $45 \mathrm{mg}$ sucrose pellet to be dispensed if the response was correct, and a 4 sec. intertrial interval to begin.

\section{Stimuli}

The stimuli consisted of luminous metric patterns formed by lighting some combination of the $16,3 / 8 \mathrm{in}$. square lights (elements) mounted adjacently in a 4 by 4 matrix (cf., method 6 described by Attneave \& Arnoult, 1956; Kennedy \& Willcutt, 1965; Webster, 1966). Although $2^{16}$ different patterns were available, those patterns which contained "holes"' (unlighted elements completely surrounded by lighted ones) or "floating elements" (lighted elements not adjacent to another lighted element by at least one corner) were discarded, leaving a potential stimulus population of 28,732 pattern stimuli. Patterns generated from 3 by 4 matrices of lights were used in the previous experiments of this series, but 4 by 4 matrices were used in the present experiment for three reasons: (1) The number of unique patterns which can be generated from a 4 by 4 matrix is greater $(28,732$ vs. 2,356$)$ so that longer and more elaborate experiments could be performed while maintaining the advantageous feature of never presenting a given stimulus pattern more than once to Ss in a given experiment; (2) more complex patterns, and hence more dissimilar pairs of patterns, would be available; and (3) the stimulus format would be square and symmetrical, thus allowing analyses of the saliency of selected segments of the total pattern.

The four stimulus dimensions identified in previous experiments were studied. Two of these dimensions concerned the relative areas of the two patterns which comprise a simultaneous discrimination problem, and the remaining two dimensions were analogous expressions of the contour or sidedness of the two patterns. The two area dimensions were Element Disparity (ED), or the difference between the number of lighted elements in the two patterns, and Element Level (EL), an expression of the total number of lighted elements in both patterns for a given level of ED. Table 1 lists the exact numbers of lighted elements in both patterns for each value of these two area dimensions. For example, all ED 2 problems were characterized by one pattern having two more lighted
Table 1. Number of lighted elements in each of the two patterns of each discrimination problem having listed values of the Element Disparity (ED) and Element Level (EL) dimensions used in the experiment.

\begin{tabular}{lccccc} 
& \multicolumn{6}{c}{ Element Disparity (ED) } \\
& 0 & 1 & 2 & 3 \\
\hline \multirow{2}{*}{ Element } & 1 & $6 \times 6$ & $6 \times 7$ & $7 \times 9$ & $6 \times 9$ \\
Level (EL) & 2 & $8 \times 8$ & $8 \times 9$ & $8 \times 10$ & $7 \times 10$ \\
\hline
\end{tabular}

elements than the other. Within ED 2, however, ED 2 [EL 1] problems consisted of patterns composed of 7 and 9 elements, the patterns in ED 2 [EL 2] problems had 8 and 10 elements, and patterns in ED 2 [EL 3] problems had 9 and 11 elements. Table 2 shows that the same scheme was used for the contour dimensions. Contour Disparity (CD) was the difference between the number of straight line segments (sides) around the periphery of each pattern divided by 2 . (It should be noted that a "side" could consist of several units of periphery.) Thus every discrimination problem of the experiment was characterized by one value of each of the four dimensions; e.g., if one pattern of a problem had 10 sides and was composed of 8 elements and the other pattern had 16 sides and was composed of 9 elements, the problem would have been designated ED 1 [EL 2$]$; CD 3 [SL 2] (cf., Tables 1 and 2).

The 15 variables selected for examination in the multidimensional analyses are listed in the left-most column of Table 3, and it can be seen that they include dimensions which have been proposed by other investigators as being related to assorted aspects of the physical characteristics of visual patterns which should relate to discriminability (see Hake, 1957). The first four dimensions have been discussed above, and the second four will be described in the Results section. The second, third, and fourth moments of area were proposed by Zusne (1965) and Michels and Zusne (1965). Attneave's " $\mathrm{D}$ " is an expression of "compactness" of a pattern (Attneave \& Arnoult, 1956, p. 468), perimeter relates to the extent of the periphery of a pattern (and differs from sidedness as used in this report), and the square of the perimeter divided by the area is another frequently investigated expression of compactness (Attneave, 1957; Attneave \& Arnoult, 1956; Silver,

Table 2. Number of sides on each of the two patterns of each discrimination problem having listed values of the Contour Disparity (CD) and Sides Level (SL) dimensions used in this experiment.

\begin{tabular}{lccccc} 
& \multicolumn{6}{c}{ Contour Disparity (CD) } \\
& & 0 & 1 & 2 & 3 \\
\hline \multirow{3}{*}{ Sides } & 1 & $8-8$ & $6-8$ & $8-12$ & $8-14$ \\
Level (SL) & 2 & $12-12$ & $10-12$ & $10-14$ & $10-16$ \\
& 3 & $16-16$ & $14-16$ & $12-16$ & $12-18$ \\
& 4 & $20-20$ & $18-20$ & $14-18$ & $14-20$ \\
\hline
\end{tabular}


Landis, \& Messick, 1966; Stenson, 1966). For each of these latter six dimensions the appropriate value was computed for each of the two patterns of a problem, and the absolute difference between these two values was used as the predictor value for that problem. (It should be added that the "number of independent turns" dimension Attneave found to be the best predictor of judged complexity of random shapes is related to the $C D$ dimension of metric patterns because a "side" has been defined here as a continuous straight line segment of the periphery. Thus the number of "sides" on a metric pattern is the same as the number of "turns" a round its periphery.)

The final dimension, Unique Elements, was added, at least initially, to determine whether Ss were not discriminating simply on the basis of total, nonconfigurational differences between the patterns, perhaps by comparing spatially corresponding elements in the two patterns. The number of Unique Elements in a problem was computed as follows. The 16 elements in each display were arbitrarily numbered 1 through 4 (left to right) for the four lights in the top row, 5-8 for the second row, etc. For a given pair of patterns, the state (lighted or not) of each correspondingly numbered element was compared (element No. 1 in one pattern compared with element No. 1 in the other pattern, No. 2 with No. 2, etc.) and one Unique Element was counted for every such pair of elements in a dissimilar state. The total number of Unique Elements in a problem was, then, simply the total number of spatially corresponding elements in different states (one lighted, one not). One rationale for examining this Unique Elements dimension was the possibility that Scould attend to only one Unique Element and totally disregard the remainder of the patterns in order to perform the discriminations flawlessly. Since it seemed unlikely that any single pair of corresponding elements would be consistently sampled on every problem by every $S$, the total number of Unique Elements in a problem was used because this number should be directly related to the probability that $\mathrm{S}$ will attend to and sample any one of the independent and completely informative Unique Elements.

\section{Experimental Design}

The four levels of ED, three levels of EL, four levels of $\mathrm{CD}$ and four levels of SL (see Tables 1 and 2) were combined factorially to yield a 192-cell matrix which was the basic experimental design for each of the eight replications of the experiment (1536 problems in all). Each cell in the basix matrix specified the numbers of elements and sides of the two patterns of a single problem, and over the eight replications, therefore, eight problems were presented at each of the 192 unique combinations of values of the four dimensions under study. It should be understood that balanced values of ED, EL, CD, and SL (Tables 1 and 2) were incorporated into the basic design of the experiment, and values of the additional 11 dimensions listed in Table 3 were
Table 3. Results of the Discriminant Analysis and Multiple Regression Analysis described in the text.

\begin{tabular}{|c|c|c|c|}
\hline \multirow[b]{2}{*}{ Dimensions } & \multirow{2}{*}{$\begin{array}{c}\begin{array}{c}\text { Discriminant } \\
\text { Analysis } \\
\text { Overall } F=14.84^{*} \\
\text { Dimensional } \\
\text { F - value }\end{array}\end{array}$} & \multicolumn{2}{|c|}{$\begin{array}{c}\text { Mult. Regression } \\
\text { Analysis } \\
\text { Multiple } R=0.46^{*}\end{array}$} \\
\hline & & $\begin{array}{c}\text { Pearson } \\
r\end{array}$ & $\begin{array}{c}\text { Partial } \\
\text { corr. coef. }\end{array}$ \\
\hline 1. ED & 0.21 & $.224 *$ & .020 \\
\hline 2. $E L$ & 0.33 & -.120 & -.012 \\
\hline 3. CD & 3.02 & .116 & .024 \\
\hline 4. SL & 0.17 & $-.227 *$ & -.061 \\
\hline 5. $E D+1 / E L$ & 0.75 & $.248 *$ & .013 \\
\hline 6. $\mathrm{CD}+1 / \mathrm{SL}$ & 1.99 & $.216 *$ & .025 \\
\hline 7. $\sqrt{\mathrm{ED}+1} / \mathrm{EL}$ & 0.52 & $.249 *$ & .030 \\
\hline 8. $\sqrt{C D+1} / S L$ & 0.53 & $.242 *$ & .007 \\
\hline 9. 2nd Moment of Areo & a 2.06 & $.192 *$ & .006 \\
\hline 10. 3rd Moment of Areo & 0.83 & -.049 & -.063 \\
\hline 11. 4th Moment of Area & 0.32 & -.030 & -.023 \\
\hline 12. Attneave's "D" & 0.18 & $.218 *$ & .006 \\
\hline 13. Perimeter (P) & 3.24 & .169 & .029 \\
\hline 14. $P^{2 / \text { Area }}$ & 1.38 & .138 & .030 \\
\hline 15. Unique Elements & $69.28 *$ & $.280 *$ & $.26]^{*}$ \\
\hline
\end{tabular}

$*=p<.001$

computed on these same 1536 problems.

The order of problem presentation was random except for several balancing procedures to provide an optimally uniform rate of exposure of all levels of each of the four basic dimensions. Additionally, to control for possible area or sidedness preferences, the larger pattern of a problem was rewarded as often as the smaller, and rewarding the pattern with more or fewer sides was similarly balanced. All Ss received the same order or problem presentation, but Ss of Group A were rewarded for responding to one of the patterns of each problem, while Group B Ss were rewarded for responding to the other pattern-a further control for possible pattern preferences. Individual patterns were drawn from the available population (appropriate number of elements and sides) randomly without replacement.

In addition to the basic design problems of the last four replications of the experiment, Ss were also presented with 48 problems composed of two identical patterns, where one of the patterns was presented displaced and/or rotated within the 4 by 4 stimulus matrix. Four types of Displacement were studied; (1) Horizontal $(\mathrm{H})$ displacement of one entire lighted pattern by one column within the 4 by 4 matrix; (2) Vertical (V) displacement of one pattern by one row; (3) $\mathrm{H}+\mathrm{V}$ displacement by one column horizontally and one row vertically; and (4) No displacement. The Rotation variable also had four values, $0^{\circ}, 90^{\circ}, 180^{\circ}$, and $270^{\circ}$. The Displacement and Rotation variables were combined factorially to produce 16 problem types, and three replications of the 16-problem matrix were presented-a total of 48 problems. Two such problems were presented every day randomly intermixed among the basic design problems, and balancing procedures similar to those described above were enforced. 


\section{Procedure}

All problems were presented for seven trials because it had been determined that these learning set-sophisticated monkeys show little additional intraproblem learning after the seventh trial of such problems (Polidora \& Thompson, 1965). Fourteen problems were presented daily (16 in the last four replications), five weekdays per week, and each $\mathrm{S}$ was fed its normal daily food ration immediately after being tested. The experiment was completed in 21 consecutive weeks.

\section{RESULTS AND DISCUSSION}

Analyses of variance of the data revealed a small but consistent and statistically significant increase in discriminative performance (percentage of correct responses on Trials $2-7$ ) across the eight replications of the experiment. But since there were no significant interactions between replications and any of the stimulus dimensions (including the Unique Elements dimension), the data from all eight replications were pooled. The Groups main effect (A vs. B) was not significant, so unless explicitly stated, the data to be presented will be based upon the performance of the entire sample of 14 Ss on all problems.

The data presented in Figure 1 show that the effects of the four previously identified dimensions were practically identical to those reported earlier when the similar but smaller patterns from the 3 by 4 matrices were used. ED and CD were directly related to discriminative performance and EL and SL were inversely related. The main effects of each of these four dimensions were statistically significant ( $p<.001$ in each case), and none of their interactions were. Comparisons of these dimensional effects upon performance with earlier Trials 2-7 data (ef., Polidora \& Thompson, 1965, Fig. 1) reveal that the slopes of the functions are highly similar, but the intercepts are uniformly increased by about $5 \%$. This general increase in discriminative performance by the same Ss is more probably due to

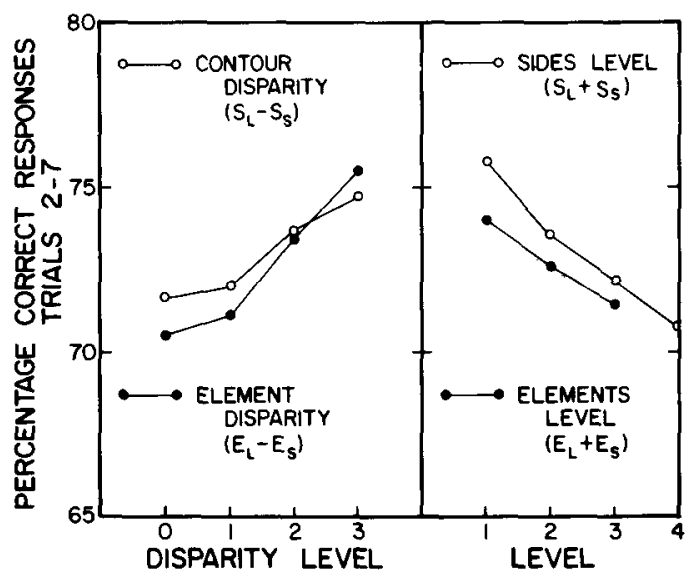

Fig. 1. Discriminative performance by the $14 \mathrm{Ss}$ as a function of the four basic dimensions, ED, EL, CD and SL.

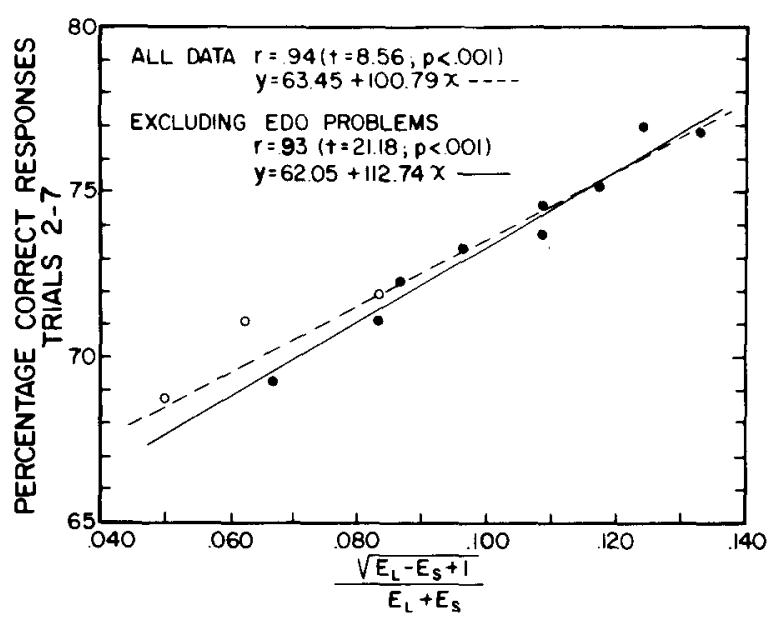

Fig. 2. Discriminative performance as a function of the area ratio dimension described in the text.

practice than to greater discriminability of patterns from 4 by 4 matrices because the dimensional functions from the first replication of the present experiment are very similar to those of the prior one, and the $5 \%$ mean increase in performance was achieved gradually in the course of the eight replications of the present experiment.

The question has been raised (Polidora, 1965b, p. 468) as to whether the "disparity" dimensions (ED and CD) and the "levels" dimensions (EL and SL) under investigation were operating as two classical psychophysical functions of the $\Delta I / I$ class. Expressed in this way the two area dimensions, ED and $E L$, would reduce to one dimension, $\mathrm{K} \cdot \mathrm{ED} / \mathrm{EL}$, or some constant times the difference in area divided by the total area of the two patterns. Expressed in terms of the number of lighted elements in larger pattern $\left(E_{L}\right)$ and the smaller pattern $\left(E_{S}\right)$, the proposed dimension would be $\mathrm{K}\left(\mathrm{E}_{\mathrm{L}}-\mathrm{E}_{\mathrm{S}}\right) / \mathrm{E}_{\mathrm{L}}+\mathrm{E}_{\mathrm{S}}$ for area, and $\mathrm{K}\left(\mathrm{S}_{\mathrm{L}}-\mathrm{S}_{\mathrm{S}}\right) / \mathrm{S}_{\mathrm{L}}+\mathrm{S}_{\mathrm{S}}$ for contour, where $S_{L}=$ pattern with the larger number of sides, $\mathrm{S}_{\mathrm{S}}=$ smaller.

The data in Figs. 2 and 3 show the relation between performance and the proposed area (Fig. 2) and contour (Fig. 3) dimensions when 1 is added to each numerator to eliminate zeros and the resulting numerator is raised to the $1 / 2$ power. The relation between dis criminative performance and these dimensions is direct, linear, and quite regular except for the departures (open circles in the figures) representing problems having two patterns with equal area (ED 0) or an equal number of sides (CD 0 ). Performance on ED 0 and CD 0 problems was better than the proposed dimensions predicted it should have been. (It should be noted that both Figs. 2 and 3 represent all the data of the experiment; in one case collapsed across the area dimensions and in the other, across the contour dimensions.)

Assuming that the best single predictor of Ss' per- 
formance on a given problem would be simply the sum of the problem's area and contour values, the graphic plot presented in Fig. 4 is obtained. In this figure each of the 192 problem classes (unique combinations of values of $\mathrm{ED}, \mathrm{EL}, \mathrm{CD}$ and $\mathrm{SL}$ ) is represented by a single point which is the mean performance by the 14 Ss on eight such problems. Again, there was a statistically significant, direct linear relation $(r=.67)$ between performance and the combined area and contour dimension, but, of course, the same departures for ED 0 and/ or CD 0 problems (open circles) were obtained.

In an attempt to identify other sources of variability of discriminative performance relating to the physical characteristics of the stimuli, particularly those responsible for the inordinately good performance on ED 0 and $C D \quad 0$ problems, several additional statistical analyses of these data were performed. Two mathematically different but conceptually related techniques were employed; discriminant analysis, and multiple regression and partial correlation analyses (Hake, 1965 and Hake \& Rodwan, 1966, discuss the application of these techniques to perceptual problems).

The first discriminant analysis of these data posed the question: What weighted linear combination of the

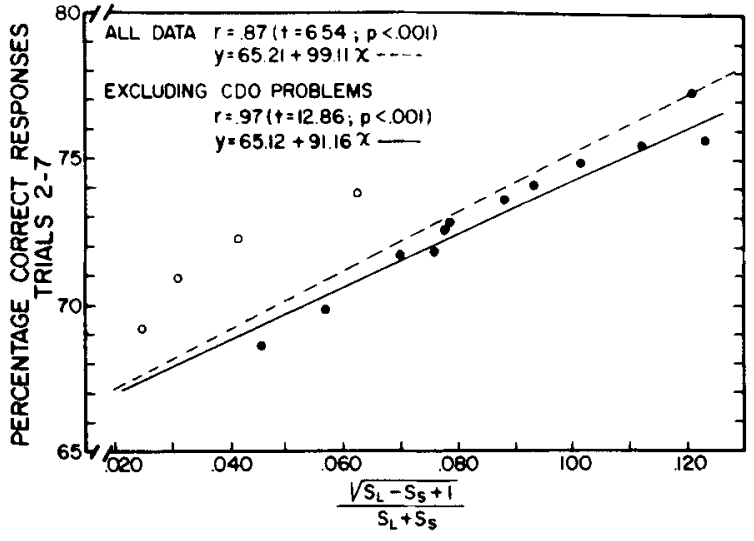

Fig. 3. Discriminative performance as a function of the contour ratio dimension described in the text.

15 dimensions best discriminates between two sets of problems upon which Ss performed approximately equally; viz., the 100 problems yielding the best performance and the 100 problems yielding the worst performance? The obtained overall F-value of 14.84 $(p<.001)$ indicated that some weighted linear combina-

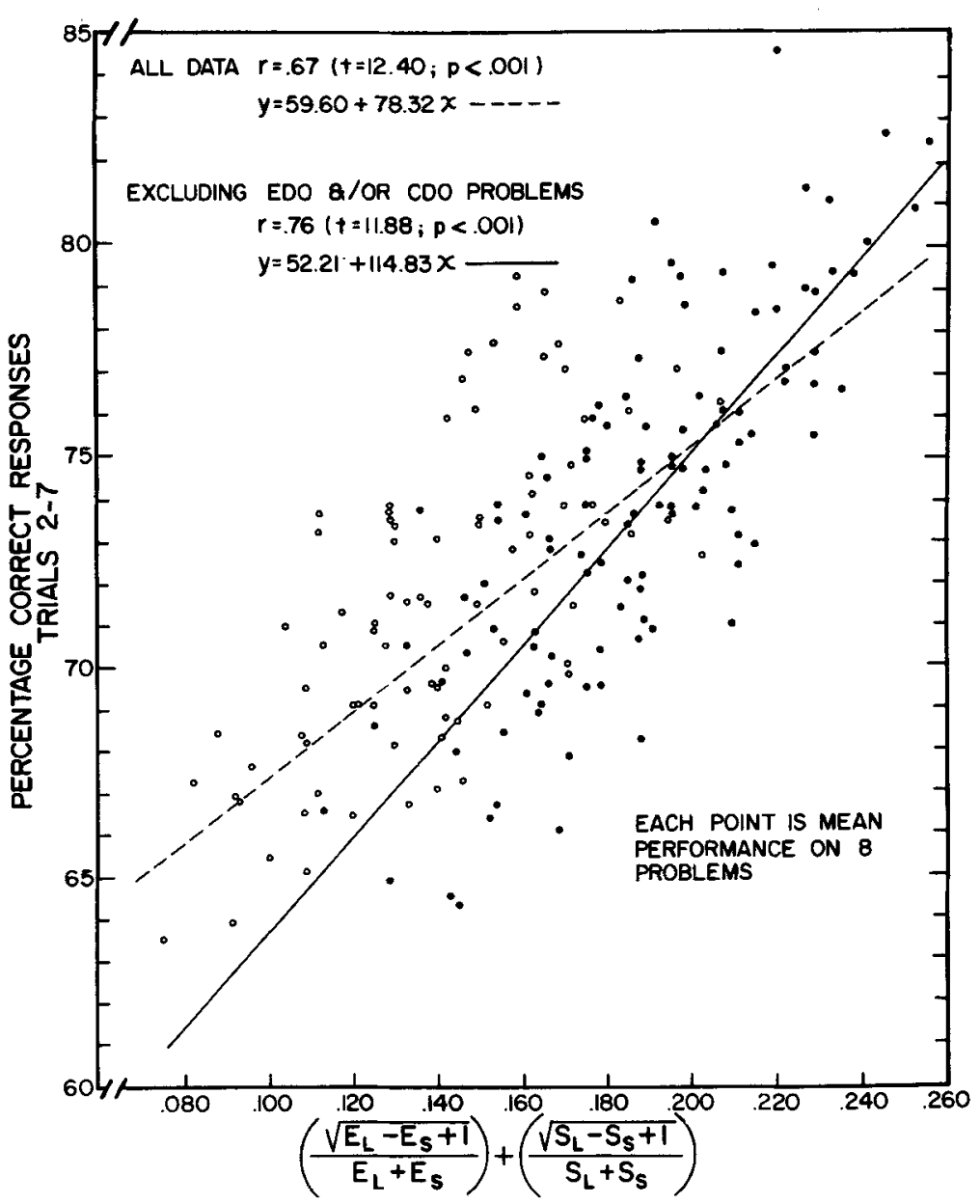

Fig. 4. Discriminative performance as a function of the combined area and contour ratio dimensions. 
tion of the 15 variables discriminated significantly well between these two populations of problems, and the F-values for each dimension (Table 3) clearly show that only one dimension, Unique Elements, determined the discriminant function. On the basis of the number of Unique Elements alone, 180 of the 200 problems were correctly identified as being easy or difficult-90\% accuracy. Several additional discriminant analyses were performed with the same dimensions and different samples of problems; easiest 500 problems (approximately $1 / 3$ of the problems) vs. the most difficult 500, successive fifths of the total sample, etc. The results of these analyses may be summarized by saying that although the accuracy with which the Unique Elements dimension predicted performance naturally decreased as the resolution requirements increased (83\% correct predictions for highest and lowest thirds of problems; $67 \%$ correct for successive fifths), Unique Elements was consistently the only statistically significant discriminant among the 15 candidate dimensions. In other words, Ss' proficiency of discrimination was predominately related to the total number of Unique Elements in a problem.

A second statistical approach to answering the same general question was provided by a multiple regression analysis performed with the same 15 dimensions as predictors of $\mathrm{Ss}^{\prime}$ performance on each of the 1536 problems. As can be seen in Table 3 , the multiple correlation obtained was a modest but statistically significant 0.46 . The individual Pearson product moment correlations between performance and the listed dimensions were uniformly low, but several of these correlation coefficients were statistically reliable. Despite the fact that discriminative performance covaried with several dimensions, which perhaps accounts for many affirmative reports of significant correlations with selected and isolated candidate dimensions, it is important to determine the extent to which intercorrelations a mong dimensions account for this effect. In computing the partial correlation between a given dimension and performance, these intercorrelations are statistically evaluated so that the resultant partial correlation coefficient expresses the degree of relationship between a given dimension and performance with the contributions of all other dimensions held constant. The right-most column of Table 3 gives these partial correlation coefficients, and these values show once again that only the Unique Elements dimension was a statistically significant predictor of performance when

Table 4.

Multiple Correlation Coefficients of Subsamples of 1536 Problems

\begin{tabular}{lccc}
$\begin{array}{l}\text { Number of } \\
\text { Problems }\end{array}$ & $\begin{array}{c}\text { Modal } \\
\text { Interval }\end{array}$ & $\begin{array}{c}\% \text { of Problems in } \\
\text { Modal Interval }\end{array}$ & Range \\
\hline $192(1 / 8$ ths $)$ & .45 to .49 & $38 \%$ & .39 to .55 \\
$96(1 / 16$ ths $)$ & .50 to .54 & $32 \%$ & .31 to .66 \\
44 (132nds) & .60 to .64 & $40 \%$ & .33 to .78 \\
\hline
\end{tabular}

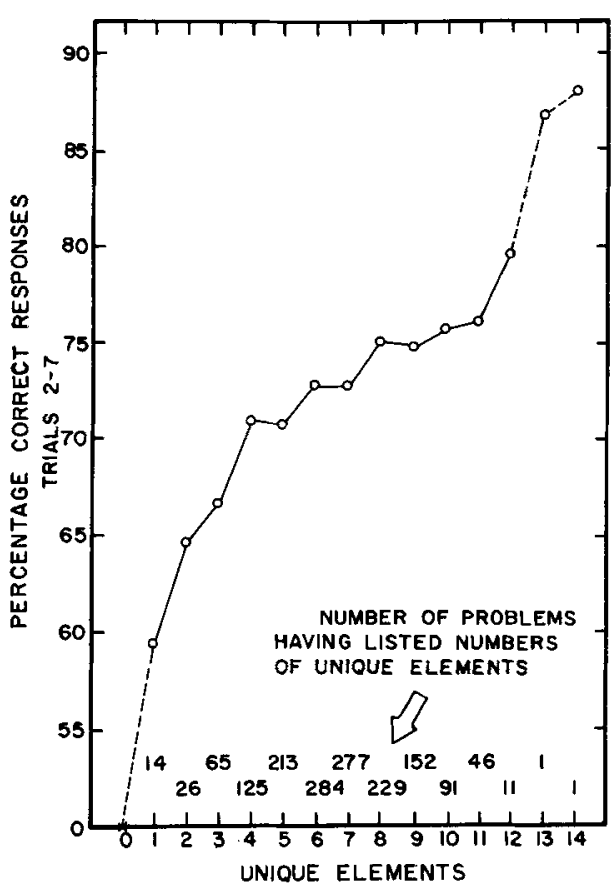

Fig. 5. Mean discriminative performance on all problems having listed numbers of Unique Elements. Note that there were unequal numbers of problems at each level of Unique Elements. The data point for zero Unique Elements is theoretical, and the data points for 13 and 14 Unique Elements are both based on only one such problem.

all other dimensions were held constant.

Thus the results of these two statistically dissimilar techniques point to the same conclusion-Unique Elements is the single most important, perhaps the only important, dimension for predicting the proficiency with which monkeys will perform on visual metric patterm discrimination problems. Moreover, these analyses illustrate the extent to which simple correlational studies in this area of research can be so misleading - witness the impressively orderly functions presented in Figs. 1 through 4, which have now been shown to be simply the result of spuriously high intercorrelations between these dimensions and the Unique Elements dimension.

Although the results of the multiple regression analysis appeared to be clear cut, the absolute magnitude of the correlation coefficients were relatively low. Since such a large number of problems (1536) was being considered, it remained to be determined whether analyses of smaller sub-samples of the total number of problems would yield similar results. Separate multiple regression analyses (otherwise identical to the initial analysis) were performed on eight randomly selected samples of 192 problems each (1/8th of the problems), then on 16 samples of 96 problems each, and finally on 32 randomiy drawn samples of 44 problems each (1/32nd of the total number of problems). The multiple correlation coefficients obtained from these analyses 
(Table 4) suggest that the large number of problems did indeed suppress the coefficients to some extent because analyses of smaller samples of the same data yielded larger coefficients. With a sample of 44 problems, for example, $40 \%$ of the multiple correlation coefficients were of the order .60 to .64 .

However, since all analyses above were performed on individual problems, there remains the further question of the extent to which obtaining statistically more stable estimates of performance at each level of Unique Elements would influence the obtained correlations. One approach to answering this question was a multiple regression analysis using as the dependent variable the mean performance of the Ss averaged across each level of Unique Elements. The multiple correlation coefficient for this analysis was .992 with the Unique Elements dimension once again having the only significant partial correlation, $.857(p<.001)$. The direct form of this relation is shown in Fig. 5. It remains to be determined whether the "true" shape of this function is exponential, as suggested by the bulk of the data, or linear, as suggested by the inordinately good performance on the relatively few problems at 12,13 and 14 Unique Elements.

Nevertheless, it must be acknowledged the results of even this latter analysis may also be somewhat suspect, although probably not too greatly in error, because there was an unequal number of problems at each level of Unique Elements (see Fig. 5). Several product moment correlations were computed between performance and the Unique Elements dimension for independently selected samples of 10,20 , and 30 problems, each randomly drawn from the total population of 1536. The results of these analyses (Table 5,

Table 5. Pearson product moment correlations between Unique Elements and mean performance of the 7 "Good," the 7 "Poor" and all $14 \mathrm{Ss}$ on five independent, random samples of 10, 20 and 30 problems each.

\begin{tabular}{|c|c|c|c|}
\hline \multirow[b]{2}{*}{ Sample \# } & \multirow[b]{2}{*}{ Good Ss } & \multicolumn{2}{|c|}{$N=10$} \\
\hline & & Poor Ss & All Ss \\
\hline 1 & $.75^{* *}$ & .59 & $.78^{* *}$ \\
\hline 2 & $.81 * *$ & $.74^{* *}$ & $.81^{* *}$ \\
\hline 3 & $.91 *$ & .05 & $.65^{* * *}$ \\
\hline 4 & $.80 * *$ & .33 & $.70 * * *$ \\
\hline \multirow[t]{2}{*}{5} & $.66 * * *$ & .24 & .56 \\
\hline & & \multicolumn{2}{|c|}{$N=20$} \\
\hline 1 & $.75^{*}$ & .18 & $.65^{*}$ \\
\hline 2 & $.80^{*}$ & $.82 *$ & $.88 *$ \\
\hline 3 & $.76 *$ & .39 & $.72 *$ \\
\hline 4 & $.89 *$ & $.75^{*}$ & $.89 *$ \\
\hline \multirow[t]{2}{*}{5} & $.85^{*}$ & .47 & $.80^{*}$ \\
\hline & & \multicolumn{2}{|c|}{$N=30$} \\
\hline 1 & $.77^{*}$ & $.61^{*}$ & $.74^{*}$ \\
\hline 2 & $.79^{*}$ & $.47 * *$ & $.74^{*}$ \\
\hline 3 & $.85^{*}$ & .35 & $.82^{*}$ \\
\hline 4 & $.78^{*}$ & $.64^{*}$ & $.77^{*}$ \\
\hline 5 & $.82 *$ & $.55^{* *}$ & $.78^{*}$ \\
\hline
\end{tabular}

Table 6. Multiple and Partial Correlations Between Performance and the Number of Unique Elements in the Quarters and Halves of the Patterns

\begin{tabular}{lcc} 
Unique Elements in: & $\begin{array}{c}\text { Partial } \\
\text { Correlation }\end{array}$ & $\begin{array}{c}\text { Multiple } \\
\text { Correlation }\end{array}$ \\
\hline Total Matrix & $.261^{*}$ & $.45^{*}$ \\
lst Quadrant & $.184^{*}$ & $.42^{*}$ \\
2nd Quadrant & $.153^{*}$ & $.41^{*}$ \\
3rd Quadrant & .075 & $.34^{*}$ \\
4th Quadrant & .094 & $.33^{*}$ \\
Upper half & $.244^{*}$ & $.44^{*}$ \\
Lower half & $.119^{* * *}$ & $.40^{*}$ \\
\hline
\end{tabular}

$* p<.001$

*** $p<.05$

right-most column) suggest that if an experimenter would study as few as 20 problems, he could expect to obtain a statistically significant direct relationship between the Unique Elements values of the problems and Ss' discriminative performance.

Because the monkeys used in the present experiment were highly practiced on these problems, however, it may be that these relationships would not hold for naive or partially experienced monkeys. Certainly the data in Table 5 indicate that the performance of the seven "poor" Ss was not so highly correlated with the Unique Elements dimension as was the performance of the seven "good" Ss. It therefore seems reasonable to expect that relatively naive monkeys will also be less sensitive to differences in Unique Elements and will produce discriminative functions with less slope. But it seems remote to expect a reverse relationship or a completely unsystematic one as long as an adequate (20-30) number of problems are studied. In any event, data from naive monkeys are being obtained in this laboratory.

The question of which segment of the total patterns was more salient (i.e., containing Unique Elements which had a greater effect upon discriminative performance) was approached by analyzing Ss' performance on all problems with respect to the number of Unique Elements in each of the four quadrants of the patterns and in the upper and lower halves. Multiple regression analyses using the same 14 dimensions plus the Unique Elements dimension calculated solely on one quadrant (i.e., 0 to 4 possible Unique Elements), or one half of the pattern, etc., revealed (Table 6) that Unique Elements in the upper halves of the patterns were more highly correlated with Ss' proficiency of discrimination than were those in the lower halves of the patterns. Considering that Ss received their rewards from receptacles mounted directly below the stimulus displays, which, if anything, should produce a bias toward the lower halves of the patterns, the effect is somewhat surprising. Despite the relative saliency of the upper halves of the patterns, however, it is clear that sampling was not restricted to the upper halves because the lower halves were also significantly $(p<.05)$ 
related to performance but to a lesser degree.

The implications of the Unique Elements interpretation forced by these data warrant critical examination at this point. This position would say in effect that the monkeys in this experiment were comparing pairs of correspondingly positioned elements in the two patterns and responding on the basis of one or more pairs of spatially corresponding elements being in different states-all this within a latency between mask response and discriminative response of less than $1 \mathrm{sec}$. This, of course, seems implausible. Nevertheless, an indirect test of this possibility was made by testing the following negative proposition: "The Ss were really responding on the basis of gross differences between patterns, perhaps some kind of noncorresponding elements differences. Further, any reasonable means of computing these differences would reveal similarly large positive correlations with discriminative proficiency."

To test this assertion the number of Unique Elements in the two patterns of all problems were determined by comparing the state (lighted or not) of element No. 1 in one pattern with the state of element No. 4 in the other pattern, No. 2 in one with No. 8 in the other, No. 3 with No. 12, etc., and in this way the total number of spatially noncorresponding elements which were in a dissimilar state were computed. This method of computing Unique Elements can be considered for the moment as one means of obtaining an estimate of the gross "differences" referred to above. That is, computing Unique Elementsas if one pattern were "rotated" $90^{\circ}$ also provides a measure of the extent to which the two patterns differ. Two additional calculations of Unique Elements were made on each of the 1536 problems; the second was computed as if one display had been "rotated" $180^{\circ}$, and the third was computed for a $270^{\circ}$ "rotation." When Ss' discriminative performance was correlated with these new and somewhat artificial dimensions, the obtained correlation coefficients indicated quite clearly that only the Unique Elements dimension computed on the basis of elements in directly corresponding spatial positions was significantly related to discriminative proficiency (the coefficients for $90^{\circ}, 180^{\circ}$ and $270^{\circ}$ were $.006, .094$ and .021 , respectively).

These and the previous results would thus support the conclusion that at least early in the new problem the monkeys were sampling first one pattern and then the other and making a very rapid discriminative response based upon parallel processing of one or more nonidentical, spatially corresponding parts of the two patterns. Later in the problem, however, Ss may have been selectively sampling only limited components of the patterns-probably only one or more of the prominant Unique Elements in the upper halves of the patterns. Separate analyses of Ss' performance on Trials 2 through 4 and Trials 5 through 7 as related to Unique Elements in either half of the matrix support this conclusion in direction, but the effect was neither large nor statistically reliable.

A second important implication of a Unique Elements interpretation of these data was examined by the subexperiment designed to study discriminative performance on problems consisting of two identical patterns, where one of the patterns was displaced or rotated within its stimulus matrix. The important point in this experiment is that most dimensions or measures of a visual pattern will yield identical difference values for two such patterns, except, of course, those measures which consider the pattern's relative location in space (e.g., 'border'" effects) or its axial orientation. For example, the dimensions examined in the present experiment (Table 3), with the sole exception of the Unique Elements dimension, would uniformly predict that two such patterns would be indiscriminable. The point is raised primarily to emphasize once again the differences between research on the perception (judged complexity, recognition, etc.) of single visual patterns and research on the discriminability of two or more patterns. The present experiment is obviously related only to the latter.

The data in Table 7 show that any displacement or rotation imposed on one of the patterns made the pair of patterns highly discriminable, and, as expected, chance behavior resulted with the no rotation, no displacement condition. The product moment correlation between discriminative performance and the Unique Elements values of these 48 problems yielded a coefficient of .81-a value well in line with the coefficients and trend indicated in Tables 4 and 5 .

The Unique Elements interpretation of visual pattern discrimination presented here is not a unique concept. Uhr (1963) discusses it as a "template matching" method of pattern recognition by computers, and Monty and Boynton (1962) termed it "T-overlap," the extent to which two patterns overlap when translated. Using random shapes, Monty and Boynton showed that human Ss used the degrees of $\mathbf{T}$-overlap between patterns in judging similarity. Furthermore, they showed that when instructed to do so, Ss could be even more proficient by basing their judgments upon "TR-overlap" - the maximal amount of overlap which can be obtained by translation plus optimal rotation of the patterns. Kennedy and Willcutt (1965) had children detect the odd one among four patterns ( 8 by 8 matrices each with 32 filled squares) and showed that response latency

Table 7. Percentage Correct Responses (Trials (2-7) on Discrimination Probløms Consisting of Two Identical Pattems. One Pattern of each Pair was Rotated or Displaced as Indicated (see text).

\begin{tabular}{llllll} 
& $0^{\circ}$ & $90^{\circ}$ & $180^{\circ}$ & $270^{\circ}$ \\
\hline \multirow{3}{*}{ Displacement } & None & 51 & 82 & 60 & 67 \\
Horizontal $(H)$ & 82 & 75 & 74 & 66 \\
Vertical $(\mathrm{V})$ & 78 & 74 & 71 & 64 \\
$\mathrm{H}+\mathrm{V}$ & 80 & 79 & 79 & 81 \\
\hline
\end{tabular}


was inversely related to the number of squares (elements) in the odd pattern which differed from those in the three identical, standard patterns. They concluded that discriminability was directly related to the total number of unique elements in the odd pattern. Pick (1965) has reported indirect evidence for a unique elements interpretation of pattern discrimination in an investigation of perceptual learning in children (see also Wohlwill, 1966). She showed that children tend to learn simultaneous (but not successive) pattern discriminations by identification of the relational differences between patterns (transformation learning), rather than by identification of the absolute characteristics of one of the patterns (prototype learning). Moreover, this result was obtained for tactual as well as for visual pattern discriminations.

Although the data of the present experiment unequivocally support a Unique Elements interpretation of visual metric pattern discriminability for monkeys, the generality of this interpretation to other classes of visual patterns and to the discriminative performance of other species remains to be determined. For example, Brown and Michels (1966) maintain that these visual metric patterns may indeed be a nonrepresentation class of patterns because they vary only in metron content (variation in magnitude of a given attribute), whereas most other classes of visual patterns vary in logon content (different types of variation or attributes) as well as metron content. While this distinction may be only a matter of degree, e.g., metric patterns can vary independently in several

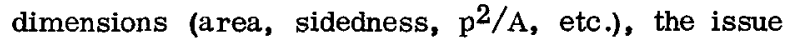
certainly warrants a test precisely because of the relative simplicity of metric patterns.

At a more theoretical level, it would appear that one of the most compelling features of a Unique Elements type of interpretation is its parsimony. For example, it could be argued that a Unique Elements or T-overlap type of explanation of visual pattern discriminability would be more a parsimonious one than those offered by Sutherland, Mackintosh, and Mackintosh (1963) to account for the increased discriminability of reduplicated patterns by octopuses, by Segstake (1965) to account for humans' speed of detecting deviations in repetitive patterns (at least for patterns of a given size), or by Woodring and Alluisi (1966) to explain the decreased ability of human Ss to correctly identify a matching metric pattern which had been rotated with respect to the nonrotated, standard pattern. Likewise, a Unique Elements type of interpretation could explain the increased discriminability of mirrorimage patterns (Riopelle et al, 1964, with line patterns and monkey Ss), or where one of the patterns has been rotated (Hunton \& Hick, 1965, with line patterns and monkey and children Ss; Hitchcock, Michels, \& Brown, 1963, with random shapes and tree squirrels and raccoons). Thus the ease with which a Unique Elements type of explanation can be applied to the results of other experiments using different classes of visual patterns and other species suggests at least limited generality of the interpretation.

Regardless of the generality of a Unique Elements interpretation, however, the present data clearly demonstrate the practical utility of using a Unique Elements dimension for predicting discriminative performance of monkeys under the present conditions. If this direct relation can be replicated under other conditions and in other laboratories, Es may exploit this relationship by using problems scaled for discriminability as an independent variable. Such a variable would be particularly useful in experiments where $\mathrm{E}$ wants to evaluate the effects of some treatment upon the range of visual discriminative ability of treated Ss.

\section{References}

Attmeave, F. Physical determinants of the judged complexity of shapes. J. exp. Psychol., 1957, 53, 221-227.

Attneave, F., \& Arnoult, M. D. The quantitative study of shape and pattern perception. Psychol. Bull., 1956, 53, 452-471.

Brown, D. R., \& Michels, K. M. Quantification procedures, stimulus domains and discriminative difficulty. Percept. mot. skills, 1966 , $22,421-422$.

Hake, H. W. Contributions of psychology to the study of pattern vision. Wright Patterson Air Force Base, Ohio: Wright Air Development Center, 1957. (WADC TR 57-621).

Hake, H. W. Multivariate methods in perception. In Cattell, R. B. (Ed.), Handbook of multivariate experimental psychology. Chicago: Rand McNally, 1965.

Hake, H. W., \& Rodwan, A. S. Perception and Recognition. In Sidowski, J. B. (Ed.), Experimental methods and instrumentation in psychology. New York: McGraw-Hill, 1966.

Harlow, H. F., Harlow, M. K., Rueping, R. R., \& Mason, W. A. Performance of infant rhesus monkeys on discrimination learning, delayed response, and discrimination learning set. J. comp. physiol. Psychol., 1960, 53, 113-121.

Hitchcock, L., Michels, K. M., \& Brown, D. R. Discrimination learning: squirrels vs. raccoons. Percept. mot. Skills, 1963, 16, 405-414.

Hunton, V. D., \& Hicks, L. H. Discrimination of figural orientation by monkeys and children. Percept. mot. Skills, 1965, 21, 55-59.

Kennedy, W. A., \& Willcutt, H. C. An eight-difficulty-level discrimination problem. Percept. mot. Skills, 1965, 20, 26.

Michels, K. M., \& Zusne, L. Metrics of visual form. Psychol. Bull., $1965,63,74-86$.

Monty, R. A., \& Boynton, R. M. Stimulus overlap and form similarity under suprathreshold conditions. Percept. mot. Skills, 1962, 14, 487-498.

Pick, Anne D. Improvement of visual and tactual form discrimination. J. exp. Psychol., 1965, 69, 331-339.

Polidora, v. J. Stimulus correlates of visual pattern discrimination by humans: area and contour. J. exp. Psychol., 1965a, 69, 221223.

Polidora, V. J. Stimulus correlates of visual pattern discrimination by monkeys: sidedness. Percept. mot. Skills, 1965b, 20, 461-469.

Polidora, V. J. A rigid, transparent manipulandum for visual displays. Percept. mot. Skills, 1965c, 21, 625-626.

Polidora, V. J., \& Fletcher, H. J. An analysis of the importance of S-R spatial contiguity for proficient primate discrimination performance. J. comp. physiol. Psychol., 1964, 57, 224-230.

Polidora, V. J., \& Main, W. T. Punched card programming and recording techniques employed in the automation of the WGTA. J. exp. Anal. Behav., 1963, 6, 599. 603.

Polidora, V. J., \& Thompson, W. J. Stimulus correlates of visual pattern discrimination by monkeys: area and contour. $J$. comp. 
physiol. Psychol., 1964, 58, 264-269.

Polidora, V. J., \& Thompson, W. J. Stimulus correlates of visual pattern discrimination by monkeys: pattern complexity. Percept. mont. Skills, 1965, 21, 71-79.

Riopelle, A. J., Rahm, U., Itoigawa, N., \& Draper, W. A. Discrimination of mirror-image patterns by rhesus monkeys. Percept. mot. Skills, 1964, 19, 383-389.

Sengstake, C. B. Perception of deviations in repetitive patterns. J. exp. Psychol., 1965, 70, 210-217.

Silver, C. A., Landis, D., \& Messick, S. Multidimensional analysis of visual form: An analysis of individual differences. Amer. $J$. Psychol., 1966, 79, 62-72.

Stenson, H. H. The physical factor structure of random forms and their judged complexity. Percept. \& Psychophys., 1966, 1, 303310

Sutherland, N. S., Mackintosh, J., \& Mackintosh, N. J. The visual discrimination of reduplicated patterns by octopus. Anim. Behav. 1963, 11, 106-110.

Uhr, L. "Pattern recognition" computers as models for form perception. Psychol. Bull., 1963, 60, 40-73.

Webster, R. B. Stimulus characteristics and effects of fill, distor- tion, and noise on pattern perception. Percept. mot. Skills, $1966,23,19-33$.

Wohlwill, J. F. Perceptual learning. Ann. Rev. Psychol., 1966, 17, 201-232.

Woodring, A. V., \& Alluisi, E. A. Effects of choice-figure rotation on the visual perception of form. Psychon. Sci., 1966, 4, 403-404.

Zusne, $\mathbf{L}$. Moments of area and of the perimeter of visual form as predictors of discrimination performance. J. exp. Psychol., 1965, $69,213-220$.

\section{Note}

1. Supported in part by NIH Grant FR-0167 and research contracts DA18-035-AMC-135(A) and DA18-035-AMC-368(A) from the U. S. Army Chemical $R$ \& $D$ Laboratories. In conducting the researeh reported herein, the investigators adhered to the "Principles of Laboratory Animal Care" as established by the National Society for Medical Research. I gratefully acknowle dge the advice and assistance of Prof. G. V. Glass on statistical matters and the technical assistance of W. J. Thompson, L. A. Link and D. A. Losey.

(Accepted for publication October $6,1966$. 